\title{
Search based Software Testing Technique for Structural Test Case Generation
}

\author{
M. S. Geetha Devasena \\ Assistant Professor, \\ Dept. of CSE \\ Sri Ramakrishna Engg. College
}

\author{
M. L. Valarmathi \\ Associate Professor \\ Dept. of CSE \\ Govt. College of Technology
}

\begin{abstract}
Software testing is an important activity in the software development life cycle and it is widely used validation approach in software industry, deployed by programmers and testers. The program with the moderate complexity cannot be tested completely. Innovative methods are needed to perform testing as a whole and unit testing in particular with minimum effort and time. Unit testing is mostly done by developers under a lot of schedule pressure since the software companies find a compromise among functionality, time to market and quality. Thus there is a need for reducing unit testing time by optimizing and automating the process. Test suite generation is an error-prone, tedious and time consuming part of unit testing. Two techniques are proposed to automatically generate test cases from the input domain using scatter search and tabu search for branch coverage criteria with respect to cyclomatic complexity measure.
\end{abstract}

\section{Keywords}

Software testing, Unit testing, Branch Coverage Criteria, tabu search, Scatter Search

\section{INTRODUCTION}

There is one famous saying that "Over testing is a Sin and Under Testing is a Crime". One of the main challenges in testing is that exhaustive testing is not possible, when to stop testing cannot be assessed and there is no way to show the absence of errors. With the increased pace of production schedules, the tremendous proliferation of software design methodologies and programming languages, and the increased size of software applications, software testing has evolved from a routine quality assurance activity into a sizable and complex challenge in terms of manageability and effectiveness. The major challenges to software testing in today's business environment are,

- Efficiency. Is the test cycle too long? How can you ensure every test is a good investment of time and money?

- Thoroughness. How can you tell when you are done testing? How can you be reasonably sure the program is bug-free?

- Resource Management. Are testing resources strategically allocated, focusing on the highest-risk elements of the software? Are the functionally central parts of the program receiving an acceptable level of testing?

In practice, unit level testing ranges from the ad hoc tests done by programmers as they are writing code to systematic white box testing, where Unit level testing is part of a every unit must be tested and documented by a QA and Test group. In either case, the tester begins with the goal of coverage, for it is the very purpose of unit level testing [1] to achieve the highest level of coverage possible. Unit testing is performed early in the development process and it is more cost-effective at locating errors. Identifying a minimum set of unit level tests to run is the greatest challenge of unit level testing. In an ideal world, every possible path of a program would be tested, accounting for all executable decisions in all possible combinations. But this is impossible when one considers the enormous number of potential paths embedded in any given program ( 2 to the power of the number of decisions). The challenge is to isolate a subset of paths that provide coverage for all testable units, and to make that subset as minimal and free of unit-level redundancies as possible. A good set of test cases is one which has a high chance of uncovering previously unknown errors and a successful test run is one that discovers these errors. To uncover all possible errors in a program, exhaustive testing is required to exercise all possible input and logical execution paths. But it is neither possible nor economically feasible. Therefore, a practical goal for software testing is to maximize the probability of finding errors using a finite number of test cases, performed in minimum time with minimum effort. A large number of testing methods developed over the last decades, designed to help the tester with the selection of appropriate test data because of the central importance of test case design for testing.

Existing test case design methods can be categorized into black-box testing and white-box testing. Black-box test cases are determined from the specification of the program under test and white-box test cases are derived from the internal structure of the software. But in both the cases. it is difficult to achieve complete automation of the test case design [4,9].

If a formal specification exists, then only black-box tests can be automated. Due to the limits of symbolic execution the tools supporting white-box tests are limited to program code instrumentation and coverage measurement. The test case design has to be performed manually. Hence the quality of test is reliant on the tester and the manual test case design is time-intensive and error prone when done manually.

\section{EXISITING SYSTEM}

\subsection{Random Test Data Generation}

Random test data generation techniques [2] select inputs randomly until useful inputs are found. This technique may fail to find test data to satisfy the requirements because information about the test requirements is not incorporated. The various disadvantages of this method are such as it is appropriate only for simple and small programs, many sets of values may lead to the same observable behavior and are thus 
redundant and the probability of selecting particular inputs that cause buggy behavior may be astronomically small.

\subsection{Static Method}

Static method generates test cases without execution of the program. It considers several constraints based on the input variables of the program under test. Static techniques have several problems in treatment of loops and resolution of computed storage locations. Also computational cost is high.

\subsection{Dynamic Method}

Dynamic test-data generation technique collects information during the execution of the program and it determines which test cases come closest to satisfying the requirement. These, test inputs are then incrementally modified until one of them satisfies the requirement. Most dynamic techniques use search based software techniques.

\subsection{Search based software testing}

Search-Based Software Engineering (SBSE) is the application of optimization techniques (OT) in solving software engineering problems. Optimization is the process of attempting to find the best possible solution amongst all those available. The percentage of application of search based techniques to software testing is $70 \%$ as shown in Figure 1.

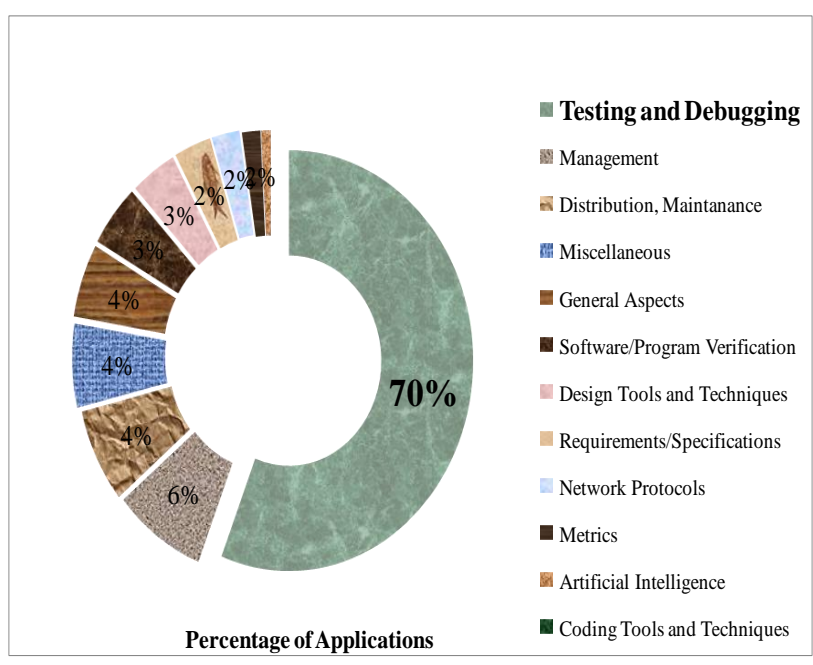

Fig 1: Application of SBSE

Software testing is a suitable candidate for Search-Based Software Engineering because the generation of software tests is an undecidable problem $[14,15]$ and a program's input space is very large, exhaustive enumeration is infeasible. To perform evolutionary testing, the task of test case design is transformed into an optimization problem and it can be solved with meta-heuristic search techniques, such as evolutionary algorithms or simulated annealing. The search space is represented by the input domain of the system under test. From this search space the test data fulfilling the test objectives under consideration is generated. The main aim of evolutionary testing is to increase the quality of the tests. Also a high degree of automation helps in cost savings in system development. In various case studies, it has been proved that evolutionary testing has the potential to improve the effectiveness and efficiency of the testing process significantly. An overview of different applications of evolutionary testing is provided by McMinn [12].

\subsection{Symbolic test case generation technique}

Symbolic test data generation techniques [7, 8] assign symbolic values to the variables and create algebraic expressions for the several constraints in the program. A constraints solver is used to find a solution for these expressions that satisfies a test requirement. This technique cannot determine which symbolic values of the potential values will be used. The constraint solvers cannot produce floating point constraints and hence floating point inputs cannot be found.

\section{STRUCTURAL TESTING \\ 3.1 Bug Statistics}

The bug statistics [17] through SDLC collected from various sources given by Boris Beizer for a program of 1,00,000 lines of code shown in table 1 , among the other bugs structural bugs are the highest and half of the structural bugs are control flow and sequence bugs as shown in Figure 2.The automated structural testing techniques can help in reducing these bugs to a large extent.

Table 1. Bug Statistics

\begin{tabular}{|c|c|c|}
\hline \multicolumn{3}{|c|}{ Size of source code: 6870000 statements } \\
\hline \multicolumn{2}{|c|}{ Total Reported Bugs: 16209 } \\
\hline Bug Categorization & $\begin{array}{c}\text { Total number } \\
\text { of bugs }\end{array}$ & $\begin{array}{c}\text { \% of bugs among } \\
\text { the total bugs }\end{array}$ \\
\hline Requirements & 1317 & 8.1 \\
\hline Features and Functionality & 2624 & 16.2 \\
\hline Structural Bugs & $\mathbf{4 0 8 2}$ & $\mathbf{2 5 . 2}$ \\
\hline Data & 3638 & 22.4 \\
\hline $\begin{array}{c}\text { Implementation and } \\
\text { Coding }\end{array}$ & 1601 & 9.9 \\
\hline Integration & 1455 & 9.0 \\
\hline $\begin{array}{c}\text { System, Software and } \\
\text { Architecture }\end{array}$ & 282 & 1.7 \\
\hline $\begin{array}{c}\text { Test Definition and } \\
\text { Execution }\end{array}$ & 447 & 2.8 \\
\hline Other, Unspecified & 763 & 4.7 \\
\hline \multicolumn{2}{|c}{} \\
\hline \multicolumn{2}{|c|}{}
\end{tabular}

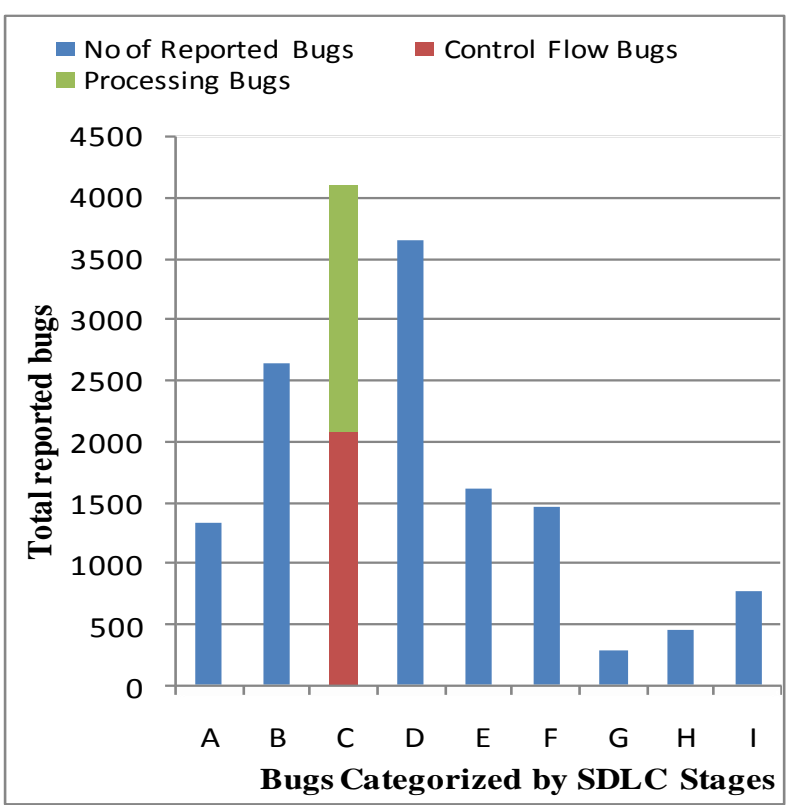

Fig 2: Bar Graph representation of Bug Statistics 
The horizontal axis details of Figure 3 is mentioned below

A-Requirements

B-Features and Functionality

C-Structural Bugs

D-Data

E-Implementation and Coding

F-Integration

G-System and software Architecture

H-Test Definition and Execution

I-Other, unspecified

\subsection{Cyclomatic complexity measure}

Cyclomatic complexity $[11,16]$ (or conditional complexity) is software structural metric (measurement) used to measure the complexity of a program using Control flow graph of the program. The cyclomatic complexity of a structured program is defined as $\mathrm{M}=\mathrm{E}-\mathrm{N}+2 \mathrm{P}$ where, $\mathrm{M}$ - Cyclomatic Complexity, $\mathrm{E}$ - the number of edges of the graph, N-The number of nodes of the graph and P-The number of disconnected components. It provides lower bound on the number of test cases required to achieve branch coverage. The amount of test effort is better judged Cyclomatic Complexity. If there are fewer test cases than the measure then missing cases are to be found and more test cases than the measure shows that the coverage can be achieved with less number of test cases.

\subsection{Evolutionary Testing}

Evolutionary testing is characterized by the use of metaheuristic search techniques for test case generation. The test aim is transformed into an optimization problem. The search space is the input domain of the test object. The search algorithm explores the search space to find test data that fulfils the respective test aim. The neighborhood search methods such as hill climbing are not suitable in such cases. So meta-heuristic search methods are employed, e.g. evolutionary algorithms, simulated annealing, or scatter search $[5,6,13]$. The robustness and suitability of evolutionary algorithms for the solution of different test tasks has already been proven in previous work [10]. But most of the previous works in applying search techniques for test case generation problem are not taking into account float values for input domain. The first work in applying scatter search to test case generation is given by Diaz and the cyclomatic complexity is not considered [3]. The proposed work extends the previous work and applies scatter search and tabu search techniques to test case generation in compliance with cyclomatic complexity measure for unit testing and compares the performance with random test case generation based on the measures of test suite size and branch coverage.

\section{PROPOSED SYSTEM}

The proposed system develops a tool for automatic test suite generation. It takes control flow graph as input and automatically generates test cases from the input domain of various variables using scatter search and tabu search techniques. The architecture of the proposed work is shown in Figure 3. The Control Flow Graph Generator takes the source code of programs for which test case is to be generated and generates Control Flow Graphs.

\subsection{Methodology}

The various steps in the automated framework of test case generation are,
1. Taking source code under test as input CFG generator generates $\mathrm{CFG}$.

2. Find the Cyclomatic Complexity measure.

3. The CFG is analyzed and the branching condition information is extracted.

4. The test cases are generated for each condition from input domain of the variables involved in the condition using scatter search technique.

5. Find the compliance of number of test cases with Cyclomatic Complexity measure.

6. The generated test cases are applied to the instrumented source code to check the branch coverage.

7. The best test cases form an effective test suite for the given source code under test.

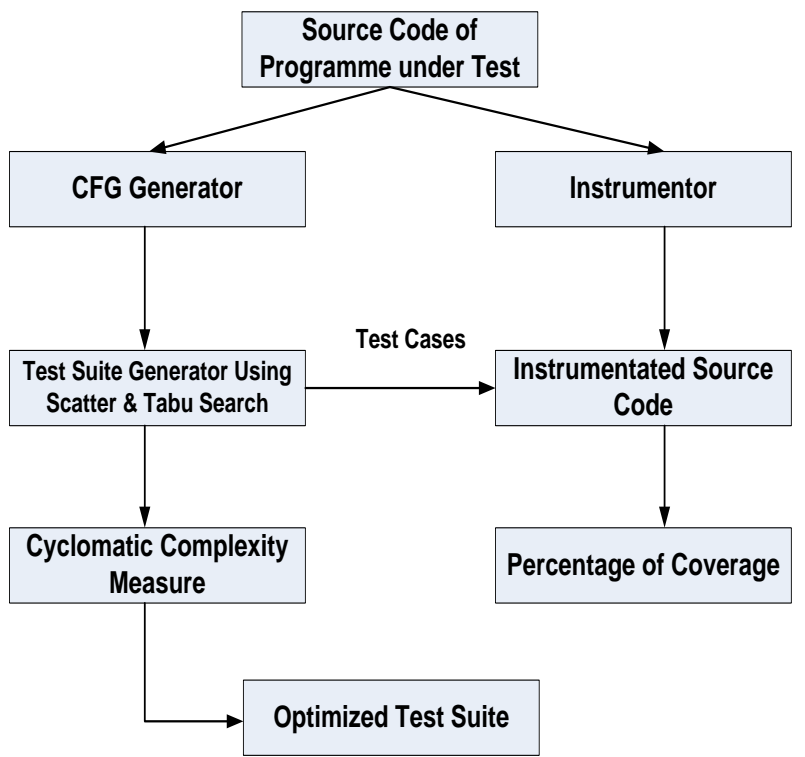

Fig 3: Flow diagram of Proposed System

Tabu search and Scatter search are search based techniques that solves a great variety of real-world problems, such as job shop scheduling, multiprocessor task scheduling, vehicle routing problems, graph coloring and many other combinatorial optimization problems. Recently it is found suitable for test case generation problems in software testing. But only few results have been published with relatively few samples and it must be further proven with all data types of input domain and with more samples. The proposed system uses Tabu and Scatter search to automate the generation of test cases to obtain high branch coverage.

\subsection{Scatter search technique algorithm}

The scatter search algorithm is given as below, begin

Initialize Current Solution

Store Current Solution in CFG

Add Current Solution to memory list do

Select a subgoal node to be covered

Calculate neighbourhood candidates

for each candidate do

calculate branch covered by candidate endfor

if (subgoal node covered) then Add Current Solution to memory list

else Add Current Solution to memory list 
endif

while (NOT all nodes covered AND number of end iterations $<$ MAXIT)

\subsection{Tabu search technique algorithm}

The Tabu search algorithm is given as below, begin

Initialise Current Solution

Store Current Solution in CFG

Add Current Solution to tabu list ST

Select a subgoal node to be covered

Calculate neighbourhood candidates

for each candidate do

if (candidate value in node $\mathrm{n}<\mathrm{CFG}$ in node $\mathrm{n}$ ) then Store candidate in $\mathrm{CFG}$

endif endfor

if (subgoal node not covered) then Add Current

Solution to tabu list ST

\section{else}

Delete tabu list ST endif

Select a subgoal node to be covered and Current solution

if (Current Solution is depleted) then

Add Current Solution to tabu list LT

Apply a backtracking process: new Current Solution and maybe new subgoal node endif

while (NOT all nodes covered AND number of iterations $<$ MAXIT)end

\section{RESULTS}

The proposed technique has been tested with 12 benchmarking samples including the triangle classifier program which is widely used in various research papers $[1,3$, 13] in the test suite generation. The results obtained are encouraging and scatter search technique performs better than random technique. The Performance measures such as the Test Suite Size, Percentage of branch coverage are considered for comparison of the techniques. Also the test suite size is compared with the cyclomatic complexity of the program structure under test which gives the measure of test cases required to cover the program. The results got by random technique can be given in Table 2 .

Table 2. Results of Random Technique

\begin{tabular}{|c|c|c|c|}
\hline Samples & $\begin{array}{c}\text { Test Suite } \\
\text { Size }\end{array}$ & $\begin{array}{c}\text { \% of Branch } \\
\text { Coverage }\end{array}$ & $\begin{array}{c}\text { Cyclomatic } \\
\text { Complexity }\end{array}$ \\
\hline S1 & 8 & 75 & 3 \\
\hline S2 & 5 & 80 & 2 \\
\hline S3 & 7 & 100 & 3 \\
\hline S4 & 3 & 100 & 2 \\
\hline S5 & 9 & 77.77 & 3 \\
\hline S6 & 11 & 81.8 & 3 \\
\hline S7 & 5 & 100 & 2 \\
\hline S8 & 6 & 100 & 3 \\
\hline S9 & 5 & 100 & 2 \\
\hline S10 & 8 & 87.5 & 3 \\
\hline S11 & 10 & 88.88 & 3 \\
\hline S12 & 15 & 93.33 & 4 \\
\hline
\end{tabular}

The results show that the branch coverage varies from $75 \%$ to a maximum of $100 \%$ and that is achieved with more number of test cases than the calculated Cyclomatic Complexity measure. The results got by scatter search technique are given in Table 3.

Table 3. Results of Scatter search Technique

\begin{tabular}{|c|c|c|c|}
\hline Samples & $\begin{array}{c}\text { Test Suite } \\
\text { Size }\end{array}$ & $\begin{array}{c}\text { \% of Branch } \\
\text { Coverage }\end{array}$ & $\begin{array}{c}\text { Cyclomatic } \\
\text { Complexity }\end{array}$ \\
\hline S1 & 3 & 100 & 3 \\
\hline S2 & 2 & 100 & 2 \\
\hline S3 & 3 & 100 & 3 \\
\hline S4 & 2 & 100 & 2 \\
\hline S5 & 3 & 100 & 3 \\
\hline S6 & 3 & 100 & 3 \\
\hline S7 & 2 & 100 & 2 \\
\hline S8 & 3 & 100 & 3 \\
\hline S9 & 2 & 100 & 2 \\
\hline S10 & 3 & 100 & 3 \\
\hline S11 & 2 & 88.88 & 3 \\
\hline S12 & 3 & 93.33 & 4 \\
\hline
\end{tabular}

It is found that branch coverage is increased by 10 percentages and test suite size is reduced by 67 percentages. It is achieved with as many numbers of test cases as calculated by Cyclomatic Complexity measure.

Table 4. Results of Tabu search Technique

\begin{tabular}{|c|c|c|c|}
\hline Samples & $\begin{array}{c}\text { Test Suite } \\
\text { Size }\end{array}$ & $\begin{array}{c}\text { \% of Branch } \\
\text { Coverage }\end{array}$ & $\begin{array}{c}\text { Cyclomatic } \\
\text { Complexity }\end{array}$ \\
\hline S1 & 3 & 100 & 3 \\
\hline S2 & 2 & 100 & 2 \\
\hline S3 & 3 & 100 & 3 \\
\hline S4 & 2 & 100 & 2 \\
\hline S5 & 3 & 100 & 3 \\
\hline S6 & 3 & 100 & 3 \\
\hline S7 & 2 & 100 & 2 \\
\hline S8 & 3 & 100 & 3 \\
\hline S9 & 2 & 100 & 2 \\
\hline S10 & 3 & 100 & 3 \\
\hline S11 & 3 & 100 & 3 \\
\hline S12 & 4 & 100 & 4 \\
\hline
\end{tabular}

The branch coverage is found to be 100 percentage in tabu search is achieved due to back tracking process as shown in Table 4. The list of sample programs under test is shown in Table 5.

Table 5. List of sample programs

\begin{tabular}{|c|l|}
\hline Sample Number & \multicolumn{1}{|c|}{ Program under test } \\
\hline S1 & Perfect square root \\
\hline S2 & Bessel \\
\hline S3 & Greater than zero or not \\
\hline S4 & Greatest of two no. \\
\hline S5 & GCD \\
\hline S6 & Sum of a number \\
\hline S7 & Factorial \\
\hline S8 & Fibonacci \\
\hline S9 & Reverse of a number \\
\hline S10 & Greatest of three number \\
\hline S11 & Prime factor \\
\hline S12 & Triangle classifier \\
\hline
\end{tabular}

The performance analysis graph based on the number of test cases in the test suite and the percentage of branch coverage of both the techniques is given in Figure 4 and Figure 5 respectively. 
Figure 6 is a snapshot of search based software testing technique

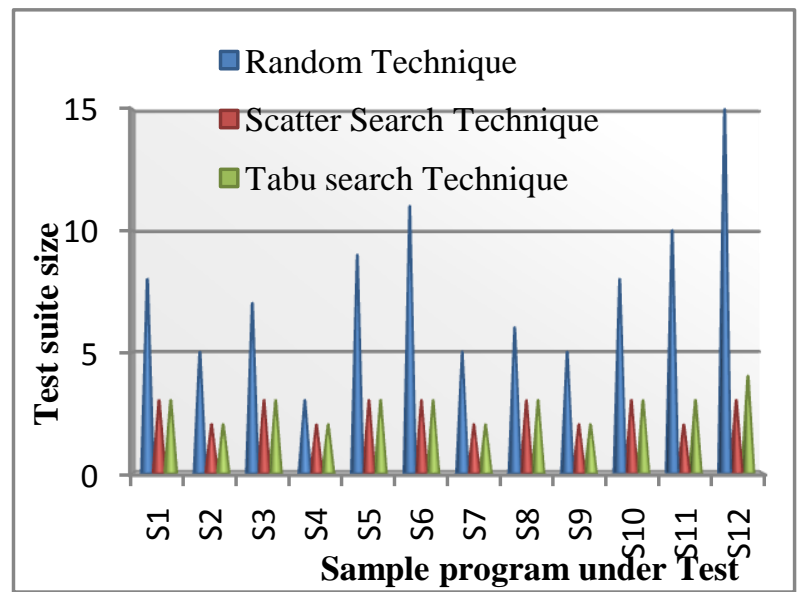

Fig 4: Test Suite Size Comparison

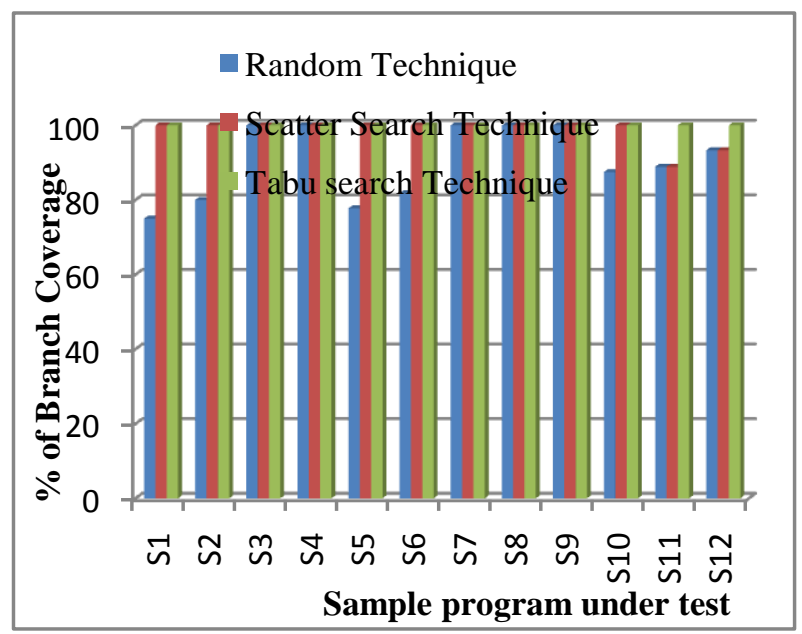

Fig 5: Percentage of Branch Coverage Comparison

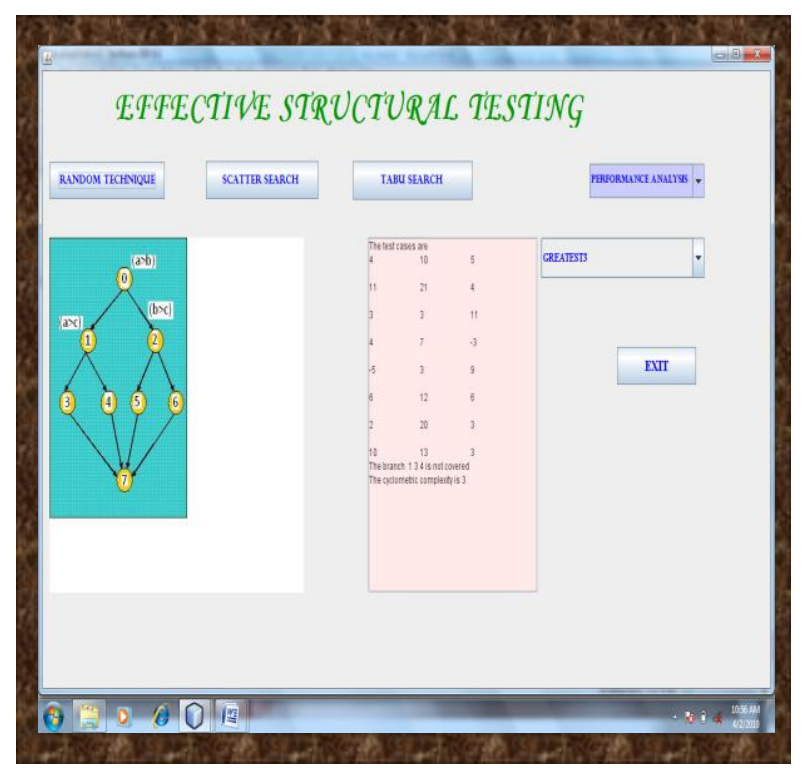

Fig 6: Snapshot of search based software testing technique

\section{CONCLUSION}

Software Testing comprises of $50 \%$ of the software development cost and also exhaustive testing is not possible. The proposed system automatically generates test cases from input domain for branch coverage criteria using Tabu search and Scatter search Techniques. Tabu search and Scatter search provides promising results and better performance than random testing,

- By reducing test suite size

- By obtaining maximum coverage

- Reducing unit testing time

- With high performance regard to range of input variables.

This technique of automated generation of test cases from the input domain can assist the developers and Quality assurance team in software companies to perform effective unit testing. Also the optimized number of test cases generated is much helpful in regression testing which otherwise carried out with greater number of test cases. This technique can be further extended for multiple coverage criteria.

\section{REFERENCES}

[1] Chilenski1, John Joseph Chilenski and Steven P. Miller, 1994. 'Applicability of Modified Condition/Decision Coverage to Software Testing', Software Engineering Journal Vol. 9, No. 5, pp.193-200.

[2] Edvardson, J. 1999. 'A Survey on Automatic Test data generation', In proceedings of the second conference on computer science and engineering Vol.2, No.1, pp.343351 .

[3] Eugenia Diaz, Javier Tuya, Raquel Blanco, Jose Javier Dolado, 2008. 'A tabu search algorithm for structural software testing', Computers and Operations Research Vol. 14, No. 3, pp.38-69.

[4] Ferguson and Korel, B. 1966. 'The chaining approach for software test data generation', ACMTOSEM vol. 5, pp.63-86.

[5] Glover, F. 1989. 'Tabu search: part I', ORSA Journal on Computing, Vol. 3, No.1,pp.190-206.

[6] Glover, F. 1990. 'Tabu search: part II', ORSA Journal on Computing, Vol. 4, No. 2, pp.4-32.

[7] Howden, W.E. 1977. 'Symbolic testing and the DISSECT symbolic evaluation system', IEEE Transactions on Software Engineering vol.3, no. 4, pp. 266-278.

[8] John Clarke, Mark Harman, Bryan Jones. 2000. 'The Application of Metaheuristic Search techniques to Problems in Software Engineering', IEEE Computer Society Press Vol.42, No.1, pp.247-254.

[9] Lindquist, T.E. and Jenkins, J.R. 1998.'Test-case generation with IOGen', IEEE Software vol.5, no.1,pp. 72-79.

[10] Lin, Yeh, P.L. 2001. 'Automatic test data generation for path testing using Gas', Information Sciences Vol. 4, No.13, pp. 47-64.

[11] McCabe, Tom, 1976. 'A Software Complexity Measure', IEEE Trans. Software Eng Vol.2, No.6, pp.308-320. 
[12] McMinn, p. 2004. 'Search Based Software Test Data Generation:A survey', Journal on Software Testing, Verification, and Reliability vol.14, no.2, pp.105-156.

[13] Raquel Blanco, Javier Tuya , Belarmino Adenso-Díaz. 2009. 'Automated test data generation using a scatter search approach', Information and Software Technology Vol. 51, No.1, pp. 708-720.

[14] Tao Feng, Kasturi Bidarkar. 2008. 'A Survey of Software Testing Methodology' vol.25, no-3, pp.216226.
[15] Voas, J.M, Morell, J. and Miller, K.W. 1991. 'Predicting where faults can hide from testing', IEEE vol: 8, pp, 41-48.

[16] Wegener, Baresel DeMillo RA, Offutt, A.J. 1991. 'Constraint-based automatic test data generation' IEEE Transactions on Software Engineering Vol.17.

[17] Boris Beizer. 2000. 'Software Testing Techniques', Second edition. 